\title{
Nuclear Enhancement of Universal Dynamics of High Parton Densities
}

\author{
H. Kowalski, ${ }^{1}$ T. Lappi, ${ }^{2}$ and R. Venugopalan ${ }^{2}$ \\ ${ }^{1}$ Deutsches Elektronen-Synchrotron DESY, 22607 Hamburg, Germany \\ ${ }^{2}$ Physics Department, Brookhaven National Laboratory, Upton, New York 11973, USA
}

(Received 5 June 2007; published 17 January 2008)

\begin{abstract}
We show that the enhancement of the saturation scale in large nuclei relative to the proton is significantly influenced by the effects of quantum evolution and the impact parameter dependence of dipole cross sections in high energy QCD. We demonstrate that there is a strong $A$ dependence in diffractive deeply inelastic scattering and discuss its sensitivity to the measurement of the recoil nucleus.
\end{abstract}

The properties of hadronic and nuclear wave functions at high energies are of great importance in understanding multiparticle production in QCD. Especially intriguing is the possibility that the small Feynman $x$ components of these wave functions demonstrate universal behavior that is insensitive to the details of hadron or nuclear structure in the (large $x$ ) fragmentation region.

The specific nature of universal small $x$ dynamics in QCD follows from the strong enhancement of gluon bremsstrahlung at small $x$ leading to a rapid growth of the occupation number of a transverse momentum mode $k_{\perp}$ in the hadron or nuclear wave function. However, it can maximally be of order $1 / \alpha_{s}$ (where $\alpha_{s}$ is the QCD coupling constant) because of nonlinear multiparton effects such as recombination and screening which deplete the gluon density at small $x$ [1]. In particular, the occupation number is maximal for modes with $k_{\perp} \lesssim Q_{s}$, where $Q_{s}(x)$, appropriately called the saturation scale, is a scale generated by the multiparton dynamics. For a probe with transverse resolution $1 / Q^{2}$, this scale is manifest in a universal scaling form of observables as a function of $Q / Q_{s}$ in a wide kinematical range in $x$ and $Q^{2}$.

In addition to the strong $x$ dependence generated by gluon bremsstrahlung, the saturation scale $Q_{s}$ has a strong $A$ dependence because of the Lorentz contraction, in the probe rest frame, of the nuclear parton density. For large enough $A$ and small enough $x$, the saturation scale is larger than $\Lambda_{\mathrm{QCD}}$, the fundamental soft scale of QCD. In this Letter, we discuss the $A$ and $x$ dependence of the saturation scale and some of its ramifications for hard diffraction in nuclei.

A saturation scale arises naturally in the color glass condensate (CGC) [2] description of universal properties of hadron and nuclear wave functions at small $x$. The CGC, when applied to deeply inelastic scattering (DIS), results [3,4], at leading order in $\alpha_{s}$, in the dipole picture of DIS [5], where the inclusive virtual photon hadron cross section is

$$
\sigma_{L, T}^{\gamma^{*} p}=\int d^{2} \mathbf{r}_{\perp} \int_{0}^{1} d z\left|\Psi_{L, T}^{\gamma^{*}}\right|^{2} \int d^{2} \mathbf{b}_{\perp} \frac{d \sigma_{\mathrm{dip}}^{p}}{d^{2} \mathbf{b}_{\perp}} .
$$

Here $\left|\Psi_{L, T}^{\gamma^{*}}\left(\mathbf{r}_{\perp}, z, Q\right)\right|^{2}$ represents the probability for a vir- tual photon to produce a quark-antiquark pair of size $r=$ $\left|\mathbf{r}_{\perp}\right|$, and $\frac{d \sigma_{\text {dip }}^{p}}{d^{2} \mathbf{b}_{\perp}}\left(\mathbf{r}_{\perp}, x, \mathbf{b}_{\perp}\right)$ denotes the dipole cross section for this pair to scatter off the target at an impact parameter $\mathbf{b}_{\perp}$. The former is well known from QED, while the latter represents the dynamics of QCD scattering at small $x$. A simple saturation model [known as the Golec-BiernatWusthoff (GBW) model [6] ] of the dipole cross section, parametrized as $\quad \frac{d \sigma_{\mathrm{dip}}^{p}}{d^{2} \mathbf{b}_{\perp}}=2\left(1-e^{-r^{2} Q_{s, p}^{2}(x) / 4}\right)$, where $Q_{s, p}^{2}(x)=\left(x_{0} / x\right)^{\lambda} \mathrm{GeV}^{2}$, gives a good qualitative fit to the HERA inclusive cross section data for $x_{0}=3 \times 10^{-4}$ and $\lambda=0.288$. However, the model does not contain the bremsstrahlung limit of perturbative QCD (pQCD) that applies to small dipoles of size $r \ll 1 / Q_{s}(x)$.

In the classical effective theory of the CGC, to leading logarithmic accuracy, one can derive the dipole cross section [4] containing the right small $r$ limit. This dipole cross section can be represented (see, however, [7]) as

$$
\frac{d \sigma_{\mathrm{dip}}^{p}}{d^{2} \mathbf{b}_{\perp}}=2\left\{1-\exp \left[-r^{2} F(x, r) T_{p}\left(\mathbf{b}_{\perp}\right)\right]\right\},
$$

where $T_{p}\left(\mathbf{b}_{\perp}\right)$ is the impact parameter profile function in the proton, normalized as $\int d^{2} \mathbf{b}_{\perp} T_{p}\left(\mathbf{b}_{\perp}\right)=1$, and $F$ is proportional to the Dokshitzer-Gribov-Lipatov-AltarelliParisi (DGLAP) evolved gluon distribution [8]

$$
F\left(x, r^{2}\right)=\pi^{2} \alpha_{s}\left(\mu_{0}^{2}+4 / r^{2}\right) x g\left(x, \mu_{0}^{2}+4 / r^{2}\right) /\left(2 N_{c}\right) .
$$

The dipole cross section in Eq. (2) was implemented in the impact parameter saturation (IPsat) model [9] where the parameters are fit to reproduce the HERA data on the inclusive structure function $F_{2}$.

In general, the dipole cross section can range from 0 in the $r \rightarrow 0$ color transparency limit to 2 , the maximal unitarity bound. The saturation scale $Q_{s}$ characterizes the qualitative change between these regimes; we here define $Q_{s}$ as the solution of $\frac{d \sigma_{\mathrm{dip}}}{d^{2} \mathbf{b}_{\perp}}\left[x, r^{2}=1 / Q_{s}^{2}\left(x, \mathbf{b}_{\perp}\right)\right]=2(1-$ $\left.e^{-1 / 4}\right)$ [10].

The IPsat dipole cross section in Eq. (2) is applicable when leading logarithms in $Q^{2}$ dominate over leading logarithms in $x$. At very small $x$, quantum evolution in 
the CGC [2] describing both the bremsstrahlung limit of linear small $x$ evolution as well as nonlinear renormalization group evolution at high parton densities, combined with a realistic $b$ dependence, is better captured in the bCGC model [11,12]. Both the IPsat model and the bCGC model provide excellent fits to a wide range of HERA data for $x \leq 0.01[12,13]$. We now discuss the possibility that DIS off nuclei can distinguish, respectively, between these "classical CGC" and "quantum CGC" motivated models.

A straightforward generalization of the dipole formalism to nuclei is to introduce the coordinates of the individual nucleons $\left\{\mathbf{b}_{\perp i}\right\}$. One obtains in the IPsat model,

$$
\frac{d \sigma_{\mathrm{dip}}^{A}}{d^{2} \mathbf{b}_{\perp}}=2\left[1-e^{-r^{2} F(x, r) \sum_{i=1}^{A} T_{p}\left(\mathbf{b}_{\perp}-\mathbf{b}_{\perp i}\right)}\right],
$$

where $F$ is defined in Eq. (3). The positions of the nucleons $\left\{\mathbf{b}_{\perp i}\right\}$ are distributed according to the Woods-Saxon distribution $T_{A}\left(\mathbf{b}_{\perp i}\right)$. We denote the average of an observable $\mathcal{O}$ over $\left\{\mathbf{b}_{\perp i}\right\}$ by $\langle\mathcal{O}\rangle_{N} \equiv \int \prod_{i=1}^{A} d^{2} \mathbf{b}_{\perp i} T_{A}\left(\mathbf{b}_{\perp i}\right) \mathcal{O}\left(\left\{\mathbf{b}_{\perp i}\right\}\right)$. The average differential dipole cross section is well approximated by [9]

$$
\left\langle\frac{d \sigma_{\mathrm{dip}}^{A}}{d^{2} \mathbf{b}_{\perp}}\right\rangle_{N} \approx 2\left[1-\left(1-\frac{T_{A}\left(\mathbf{b}_{\perp}\right)}{2} \sigma_{\mathrm{dip}}^{p}\right)^{A}\right],
$$

where, for large $A$, the expression in parentheses can be replaced by $\exp \left(-\frac{A T_{A}\left(\mathbf{b}_{\perp}\right)}{2} \sigma_{\text {dip }}^{p}\right)$ [14]. All parameters of the model come from either fits of the model to $e p$ data or from the Woods-Saxon distributions; no additional parameters are introduced for $e A$ collisions. The same exercise is repeated for the bCGC model.

In Fig. 1 (left), we compare the prediction of the IPsat and bCGC models with the experimental data [15] on nuclear DIS from the New Muon Collaboration (NMC) [16]. Figure 1 (right) shows that the $x$ dependence of shadowing for fixed $Q^{2}$ in the IPsat model is very flat.

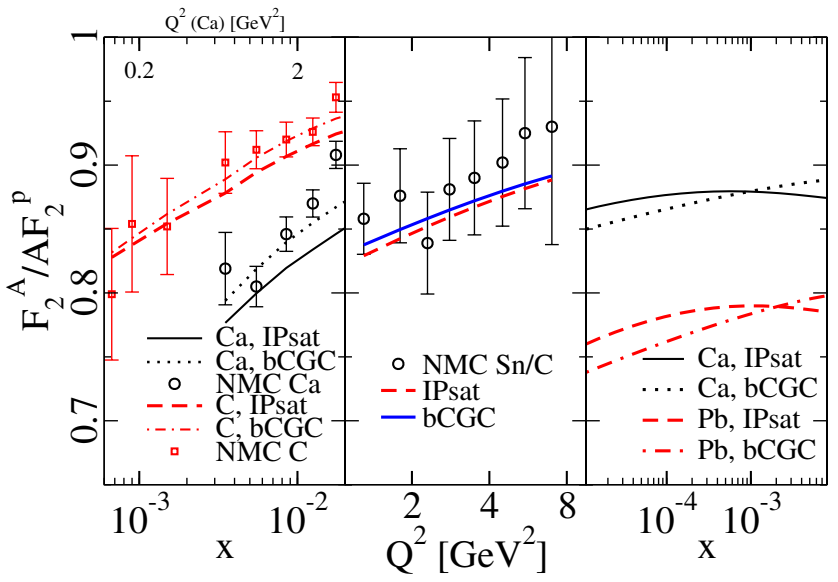

FIG. 1 (color online). Left: predictions for shadowing compared to NMC data. Center: predictions for $12 F_{2}^{\mathrm{Sn}} / 118 F_{2}^{\mathrm{C}}$ compared to NMC data at $x=0.0125$. Right: likewise for $Q^{2}=5 \mathrm{GeV}^{2}$ as a function of $x$.
This is because the best fit to $e p$ data in DGLAP-based dipole models $[8,9]$ is given by a very weak $x$ dependence at the initial scale $\mu_{0}^{2}$. A stronger $x$ dependence also for large dipoles, such as in the GBW or bCGC models, gives a stronger $x$ dependence of shadowing at fixed $Q^{2}$. As shown in Fig. 1 (center), both the IPsat and bCGC models predict strong $Q^{2}$ dependence (at fixed $x$ ) for shadowing. It is this latter effect which is primarily responsible for the shadowing effect seen in the NMC data. Precision measurements of $F_{2}^{A} / A F_{2}^{p}$ would shed more light on the relative importance of $Q^{2}$ and $x$ evolution in this regime.

We now turn to a discussion of the $A$ and $x$ dependence of the saturation scale. In a simple GBW-type model, inserting a $\theta$-function impact parameter dependence into Eq. (5) yields the estimate $Q_{s, A}^{2} \approx A^{1 / 3} \frac{R_{p}^{2} A^{2 / 3}}{R_{A}^{2}} Q_{s, p}^{2} \approx$ $0.26 A^{1 / 3} Q_{s, p}^{2}$ for $2 \pi R_{p}^{2} \approx 20 \mathrm{mb}$ and $R_{A} \approx 1.1 A^{1 / 3} \mathrm{fm}$. The smallness of $Q_{s, A}^{2} / Q_{s, p}^{2}$, due to the constant factor $\sim 0.26$ has sometimes been interpreted $[9,17,18]$ as a weak nuclear enhancement of $Q_{s}$. We argue here that detailed considerations of QCD evolution and the $b$ dependence of the dipole cross section result in a significantly larger nuclear enhancement of $Q_{s}$.

The effect of QCD evolution on $Q_{s, A}$ in the IPsat nuclear dipole cross section is from the DGLAP-like growth of the gluon distribution. The increase in the gluon density with increasing $Q^{2}$ and decreasing (dominant) dipole radius $r$ causes $Q_{s}$ to grow even faster as a function of $A$. This is seen qualitatively for two different nuclei, $A$ and $B$ (with $A>B$ ), in a "smooth nucleus" approximation of Eq. (4) whereby $\sum_{i=1}^{A} T_{p}\left(\mathbf{b}_{\perp}-\mathbf{b}_{\perp i}\right)$ is replaced by $A T_{A}\left(\mathbf{b}_{\perp}\right)$. We obtain

$$
\frac{Q_{s, A}^{2}}{Q_{s, B}^{2}}=\frac{A}{B} \frac{T_{A}\left(\mathbf{b}_{\perp}\right)}{T_{B}\left(\mathbf{b}_{\perp}\right)} \frac{F\left(x, Q_{s, A}^{2}\right)}{F\left(x, Q_{s, B}^{2}\right)} \sim \frac{A^{1 / 3}}{B^{1 / 3}} \frac{F\left(x, Q_{s, A}^{2}\right)}{F\left(x, Q_{s, B}^{2}\right)} .
$$

The scaling violations in $F$ imply that, as observed in Refs. [9,19], the growth of $Q_{s}$ is faster than $A^{1 / 3}$. Also, because the increase of $F$ with $Q^{2}$ is faster for smaller $x$, the $A$ dependence of $Q_{s}$ is stronger for higher energies. In contrast, the dipole cross section in the bCGC model depends only on the "geometrical scaling" combination [20] $r Q_{s}(x)$ without DGLAP scaling violations and therefore does not have this particular nuclear enhancement [22]. Precise extraction of the $A$ dependence of $Q_{s}$ will play an important role in distinguishing between "classical" and "quantum" evolution in the CGC.

A careful evaluation shows that because the density profile in a nucleus is more uniform than that of the proton, the saturation scales in nuclei decrease more slowly with $b$ than in the proton. The dependence of the saturation scale on the impact parameter is plotted in Fig. 2. The saturation scale in gold nuclei at the median impact parameter for the total cross section $b_{\text {med. }}$ is about $70 \%$ of the value at $b=0$; in contrast, $Q_{s, p}^{2}\left(b_{\text {med. }}\right)$ is only $\sim 35 \%$ of the value at $b=0$.

The $A$ dependence of the saturation scale for various $x$ is shown in Fig. 3, for the IPsat model on the left and the 


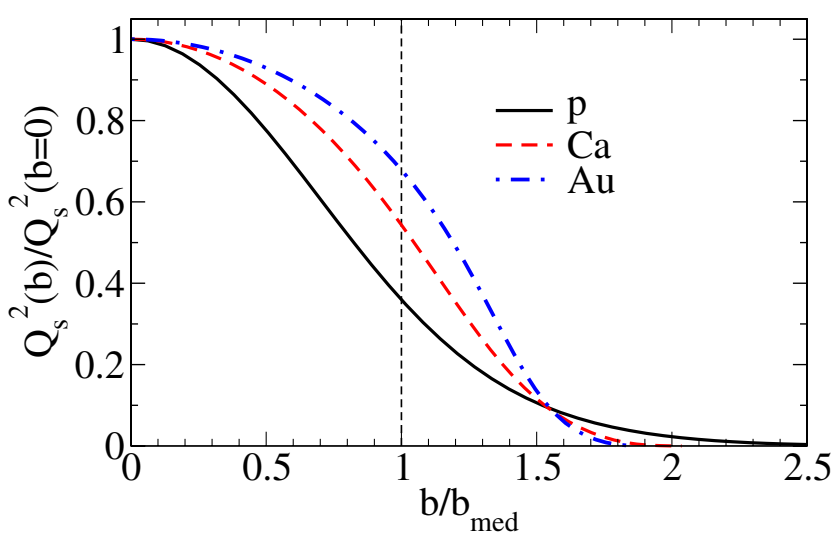

FIG. 2 (color online). Impact parameter dependence of the saturation scale for $p, \mathrm{Ca}$ and $\mathrm{Au}$ at $x=0.001$, and $Q^{2}=$ $1 \mathrm{GeV}^{2}$. Details are in the text.

bCGC model on the right. Note that in the IPsat model, at small $x, Q_{s, A}^{2}\left(b_{\text {med. }}\right)$ for gold nuclei is nearly identical to $A^{1 / 3}$ times the value for the proton. The corresponding enhancement for $b=0$ is significantly smaller as anticipated. The nuclear enhancement in the bCGC model is nearly as large, showing that it owes, for the kinematic range studied, much more to the relative impact parameter profiles (see Fig. 2) than to differences in QCD evolution. Nevertheless, the stronger $A$ dependence of $Q_{s, A}^{2}\left(b_{\text {med. }}\right)$ in the IPsat model relative to the bCGC model, especially at the smallest $x$ values, clearly illustrates the differences in quantum evolution between the models. The factor of $200^{1 / 3} \approx 6$ gives a huge "oomph" in the parton density of a nucleus relative to that of a proton; one requires a center of mass energy $\sim 14$ times larger in an $e+p$ collider relative to an $e+\mathrm{Au}$ collider to obtain the same $Q_{s, A}^{2}\left(b_{\text {med. }}\right)(x)$.

We now focus on some interesting qualitative features of hard diffraction off nuclei (see also [24]). For simplicity, we consider only the IPsat model here. The contribution of $q \bar{q}$ dipoles [25] to the inclusive diffractive cross section can be expressed as

$$
\frac{d \sigma_{L, T}^{D}}{d t}=\frac{1}{16 \pi} \int d^{2} \mathbf{r}_{\perp} d z\left|\Psi_{L, T}^{\gamma^{*}}\right|^{2} \sigma_{\mathrm{dip}}^{2}\left(x, r, \boldsymbol{\Delta}_{\perp}\right),
$$

where $t=-\boldsymbol{\Delta}_{\perp}^{2}$ and $\sigma_{\text {dip }}\left(x, r, \Delta_{\perp}\right)$ is the Fourier trans-

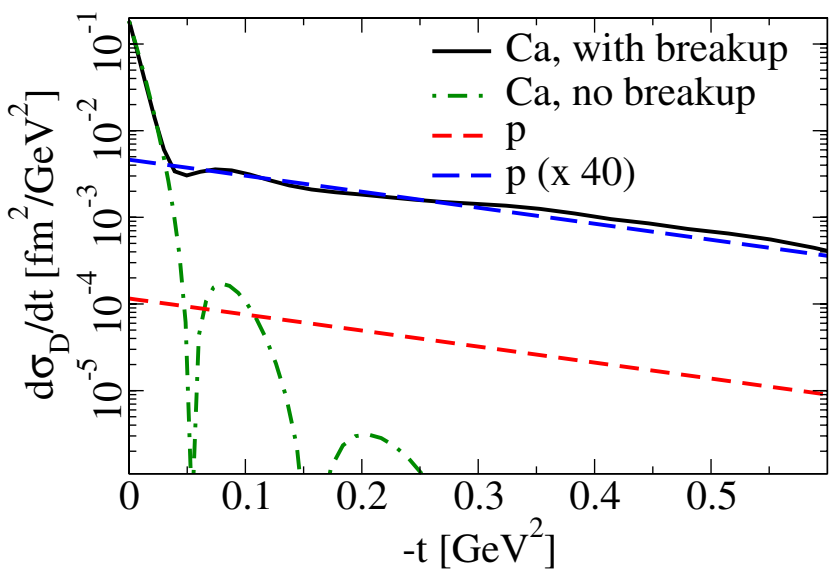

FIG. 4 (color online). The $t$ dependence of the calcium and the proton dipole cross sections. The "breakup" curve for calcium is computed using $\frac{1}{16 \pi}\left\langle\left|\sigma_{\text {dip }}\left(x, r, \Delta_{\perp}\right)\right|^{2}\right\rangle_{N}$ for $r=0.2 \mathrm{fm}$ and $x=$ 0.001 and "no breakup" curve using $\frac{1}{16 \pi}\left\langle\sigma_{\text {dip }}\left(x, r, \Delta_{\perp}\right)\right\rangle_{N}^{2}$.

form of the dipole cross section with respect to $\mathbf{b}_{\perp}$. The total diffractive cross section, obtained by integrating Eq. (7) over $t$, reads [26]

$$
\sigma_{L, T}^{D}=\frac{1}{4} \int d^{2} \mathbf{r}_{\perp} d z\left|\Psi_{L, T}^{\gamma^{*}}\right|^{2} \int d^{2} \mathbf{b}_{\perp}\left(\frac{d \sigma_{\mathrm{dip}}}{d^{2} \mathbf{b}_{\perp}}\right)^{2}
$$

The diffractive slope at $t=0$ depends on the size of the system. For small $t \sim-1 / R_{A}^{2}$ one expects a very steep $t$ dependence $\sim \exp \left\{D t R_{A}^{2}\right\}$ (with $D \sim 1$ ). In our picture of the nucleus as a "lumpy" collection of partially overlapping nucleons [Eqs. (4) and (5)], an interesting question is whether this lumpiness shows up as a protonlike tail $\left.\sim \exp \left\{D^{\prime} t R_{p}^{2}\right)\right\}$ of the $t$ distribution.

If one requires that the nucleus stays completely intact, the average $\langle\cdot\rangle_{N}$ must be performed at the amplitude level, and $d \sigma^{D} / d t$ falls off very rapidly as $\sim \exp \left\{D t R_{A}^{2}\right\}$. Measuring the intact recoil nucleus at such a small $t$ experimentally at a future electron ion collider [28] is challenging. Considerable physical insight into the diffractive process can be obtained in events where the nucleus breaks up into color neutral constituents without filling the rapidity gap between the $q \bar{q}$ dipole and the nuclear fragmentation region. Such events correspond to performing the average $\langle\cdot\rangle_{N}$ over the cross section [29], Eq. (8), instead

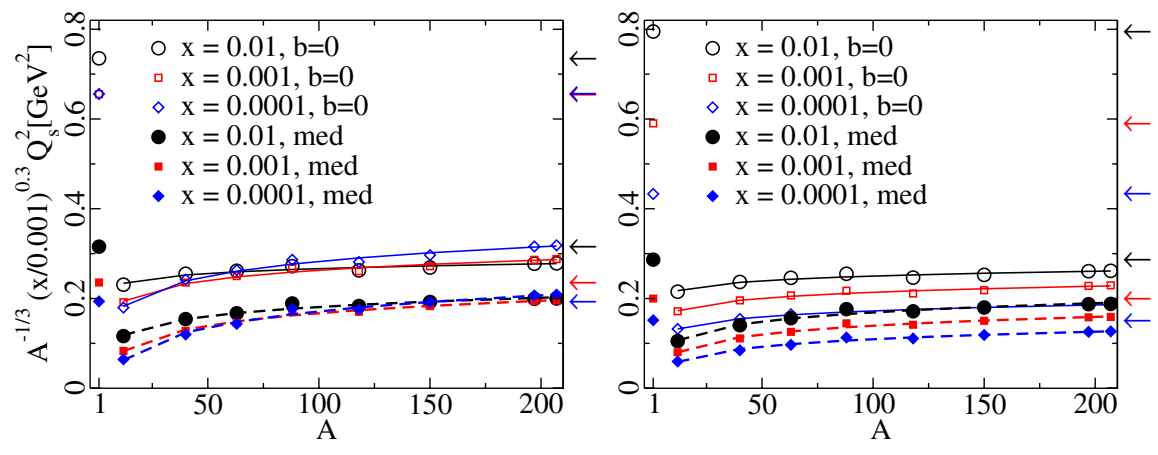

022303-3
FIG. 3 (color online). Saturation scale at $b=0$ (open symbols) and $b=b_{\text {med. }}$ (filled symbols) as a function of $A$ for different $x$. The saturation scale for the proton is shown at $A=1$ and by the arrows on the right. Left: IPsat model. Right: bCGC model. 


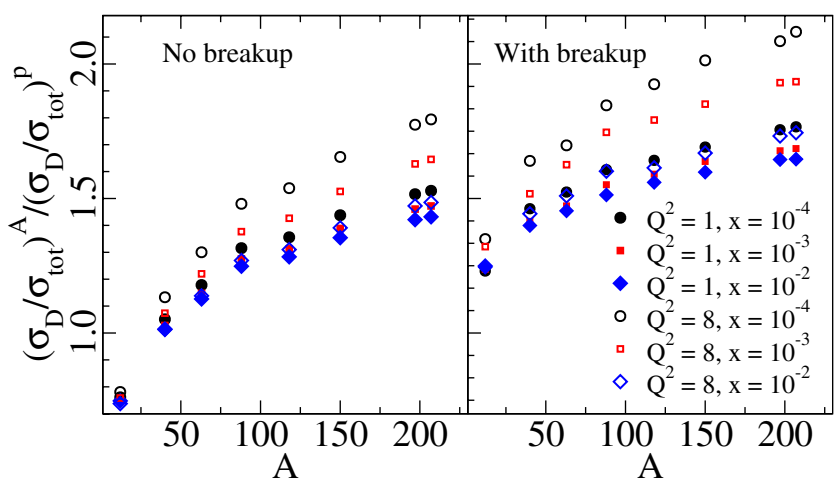

FIG. 5 (color online). Ratio of the fraction of $q \bar{q}$ diffractive events in nuclei to the fraction in a proton plotted versus atomic number $A$ for fixed $x$ and $Q^{2}$ values.

of the amplitude. The difference between the two averaging procedures can be significant with increasing values of $t$; the result for calcium nuclei is shown in Fig. 4. The $t$ dependence of the proton is shown as well; as expected, the "breakup" cross section for calcium converges to $A$ times the proton cross section with increasing $t$.

The difference between "no breakup" and breakup integrated cross sections can be seen in Fig. 5 where we plot, as a function of $A$ for fixed $x$ and $Q^{2}$, the double ratio $R_{\text {diff. }}^{A}$, defined as the ratio $\left(\sigma_{T}^{D}+\sigma_{L}^{D}\right) /\left(\sigma_{T}^{\gamma^{*}}+\sigma_{L}^{\gamma^{*}}\right)$ from Eqs. (1) and (8) for a nucleus divided by the same ratio for a proton. For light nuclei $(A<40), R_{\text {diff. }}^{A}<1$ before going well above unity for large $A$. This is because the diffractive $q \bar{q}$ cross section is dominated by smaller impact parameters than the inclusive cross section; at small impact parameters, the matter density in a proton is larger than in light nuclei. For large nuclei, especially in the breakup case, the nuclear enhancement of the fraction of diffractive events can be quite significant, up to a $100 \%$ enhancement relative to the fraction for a proton.

We thank A. Caldwell, C. Marquet, and L. Motyka for very useful discussions. This manuscript has been authorized under Contract No. DE-AC02-98CH10886 with the U.S. Department of Energy.

[1] L. V. Gribov, E. M. Levin, and M. G. Ryskin, Phys. Rep. 100, 1 (1983); A. H. Mueller and J.-W. Qiu, Nucl. Phys. B268, 427 (1986).

[2] E. Iancu and R. Venugopalan, arXiv:hep-ph/0303204; H. Weigert, Prog. Part. Nucl. Phys. 55, 461 (2005).

[3] L. D. McLerran and R. Venugopalan, Phys. Rev. D 59, 094002 (1999); Y. V. Kovchegov, Phys. Rev. D 60, 034008 (1999); E. Iancu and A. H. Mueller, Nucl. Phys. A730, 460 (2004).

[4] R. Venugopalan, Acta Phys. Pol. B 30, 3731 (1999).

[5] A. H. Mueller, Nucl. Phys. B335, 115 (1990); N. N. Nikolaev and B. G. Zakharov, Phys. Lett. B 260, 414 (1991); Z. Phys. C 49, 607 (1991); 53, 331 (1992).

[6] K. Golec-Biernat and M. Wusthoff, Phys. Rev. D 59, 014017 (1998); 60, 114023 (1999).
[7] K. J. Golec-Biernat and A. M. Stasto, Nucl. Phys. B668, 345 (2003).

[8] J. Bartels, K. Golec-Biernat, and H. Kowalski, Phys. Rev. D 66, 014001 (2002).

[9] H. Kowalski and D. Teaney, Phys. Rev. D 68, 114005 (2003).

[10] Our definition is equivalent to the saturation scale in the GBW model for a Gaussian dipole cross section. Note the difference with the convention in Ref. [9].

[11] E. Iancu, K. Itakura, and S. Munier, Phys. Lett. B 590, 199 (2004).

[12] H. Kowalski, L. Motyka, and G. Watt, Phys. Rev. D 74, 074016 (2006).

[13] J. R. Forshaw, R. Sandapen, and G. Shaw, J. High Energy Phys. 11 (2006) 025.

[14] E. Gotsman, E. Levin, M. Lublinsky, U. Maor, and K. Tuchin, Phys. Lett. B 492, 47 (2000).

[15] Data available from the E665 Collaboration differ from the NMC data for a similar kinematic range.

[16] P. Amaudruz et al. (New Muon Collaboration), Nucl. Phys. B441, 3 (1995).

[17] A. Freund, K. Rummukainen, H. Weigert, and A. Schafer, Phys. Rev. Lett. 90, 222002 (2003).

[18] B. Z. Kopeliovich, B. Povh, and I. Schmidt, Nucl. Phys. A782, 24 (2007).

[19] N. Armesto, C. A. Salgado, and U. A. Wiedemann, Phys. Rev. Lett. 94, 022002 (2005).

[20] See, however, Ref. [21] on "preasymptotic" violations of geometrical scaling.

[21] J.L. Albacete and Y. V. Kovchegov, Phys. Rev. D 75, 125021 (2007).

[22] Another interesting possibility is that running coupling $\log x$ evolution results in a depletion of the $A$ dependence of $Q_{s}$ [23].

[23] A. H. Mueller, Nucl. Phys. A724, 223 (2003).

[24] N. N. Nikolaev, B. G. Zakharov, and V. R. Zoller, Z. Phys. A 351, 435 (1995); L. Frankfurt, V. Guzey, and M. Strikman, Phys. Lett. B 586, 41 (2004).

[25] The contribution of higher Fock states will be addressed in a future work.

[26] A comparison of Eqs. (1) and (8) makes explicit the unitarity limit $\sigma_{L, T}^{D} \leq \sigma_{L, T} / 2$, which is saturated by a black disk, $\frac{d \sigma_{\text {dip }}}{d^{2} \mathbf{b}_{\perp}}\left(\mathbf{b}_{\perp}\right)=2 \theta(R-b)$, leading to the $t$ distribution $\sim 4\left|J_{1}(\sqrt{-t} R)\right|^{2} /\left(-t R^{2}\right) \approx 1+t R^{2} / 4+\mathcal{O}\left(t^{2}\right)$. In contrast, an exponential $t$ distribution $\sim e^{B t}$ (see, e.g., [27]) corresponds to a Gaussian in $b$, where the unitarity limit, saturated by $\frac{d \sigma_{\text {dip }}}{d^{2} \mathbf{b}_{\perp}}=2 e^{-b^{2} /(2 B)}$, is $\sigma_{L, T}^{D} \leq \sigma_{L, T} / 4$. Specifying the $\mathbf{b}_{\perp}$ dependence of the dipole cross section simultaneously fixes both the normalization $\sigma_{0}$ of the cross section and the diffractive slope $B=$ $d \ln \sigma^{D} /\left.d t\right|_{t=0} . \quad\left(\sigma_{0} / B=8 \pi\right.$ and $\sigma_{0} / B=4 \pi$ for black disk and Gaussian, respectively.)

[27] M. S. Kugeratski, V. P. Goncalves, and F. S. Navarra, Eur. Phys. J. C 46, 413 (2006).

[28] A. Deshpande, R. Milner, R. Venugopalan, and W. Vogelsang, Annu. Rev. Nucl. Part. Sci. 55, 165 (2005).

[29] Y. V. Kovchegov and L. D. McLerran, Phys. Rev. D 60, 054025 (1999); A. Kovner and U. A. Wiedemann, Phys. Rev. D 64, 114002 (2001). 